\title{
W-alcançabilidade para sistemas lineares com saltos markovianos a tempo discreto*
}

\author{
Daniel Gutierrez, Eduardo F. Costa, \\ Instituto de Ciências Matemáticas e de Computação, ICMC, USP, \\ 13566-590, São Carlos, SP. \\ E-mail: dgutip@icmc.usp.br, efcosta@icmc.usp.br.
}

\begin{abstract}
Resumo: Na teoria de sistemas dinâmicos lineares existem testes simples para diversos conceitos estruturais, como alcançabilidade. Testes semelhantes existem para sistemas lineares com saltos markovianos, inclusive para a denominada alcançabilidade fraca. Entretando, a demonstração da relação do teste com o conceito de alcançabilidade fraca foi elaborada explorando relações indiretas de dualidade com uma noção de observabilidade. Neste trabalho apresentamos uma demonstração direta utilizando ferramentas de álgebra linear. Além disso, apresentamos a formalização de dois problemas de controle do tipo alcançabilidade/controlabilidade e levantamos algumas questões em aberto relacionadas à busca de soluçôes para estes problemas, envolvendo a noção de alcançabilidade fraca. Incluimos um exemplo numérico ilustrativo.
\end{abstract}

Palavras-chave: Sistemas dinâmicos lineares, Sistemas com saltos markovianos, Estrutura de sistemas dinâmicos, Alcançabilidade, Controlabilidade.

\section{Introdução}

Em termos gerais, os problemas de controle envolvem modelos matemáticos que avaliam o comportamento de um sistema com objetivo de encontrar uma ação de controle adequada (com rendimento satisfatório), possivelmente levando em conta alterações do sistema. Um dos problemas mais simples e bem conhecidos trata de um modelo linear a tempo discreto, denotado por $(A, B)$, e descrito por:

$$
\left\{\begin{array}{cl}
x(k+1) & =A x(k)+B u(k), k \geq 0, \\
x(0) & =x_{0} .
\end{array}\right.
$$

Um ponto $x=x_{0}$ é chamado de controlável sempre que exista uma estratégia de controle $u=(u(0), u(1), \ldots)$ que permita levar este ponto para o origem num tempo finito. Dizemos que o par $(A, B)$ é controlável quando qualquer ponto $x \in \mathbb{R}^{n}$ é controlável. Uma condição que verifica este conceito consiste em testar se a matriz de alcançabilidade tem posto completo [3, 2$]$.

A simplicidade dos sistemas lineares determinísticos como aquele em (1) pode torná-los pouco válidos em diversas situações em que haja alterações na dinâmica do sistema. De fato, existe uma variedade de sistemas na indústria que são vulneráveis a mudanças abruptas de falhas ou perturbações. Um modelo que é satisfatoriamente usado em tais casos são os sistemas lineares com saltos markovianos (SLSM), denotado por $(\mathbb{A}, \mathbb{B})$, considerando $T=\{1,2, \ldots, N\}$ definimos por $\mathbb{A}=\left\{A_{i}\right\}_{i \in T}$ e $\mathbb{B}=\left\{B_{i}\right\}_{i \in T}$ às coleções de matrizes conhecidas a priori das quais são "escolhidas" a cada instante de tempo $k$ um par $\left(A_{\theta(k)}, B_{\theta(k)}\right)$ sendo $\theta(k) \in T$ o valor que a cadeia de Markov assume naquele instante. Para estes sistemas, também é de interesse estudar conceitos estruturais como por exemplo observabilidade, detetabilidade, estabilizabilidade, controlabilidade e alcançabilidade, como podemos ver em [3]. Estes conceitos são de muita importância, já que caracterizam a estrutura do modelo e trazem informação relevante

\footnotetext{
*Agradecimentos a FAPESP, CAPES e CNPq pelos apoios financeiros.
} 
para problemas de filtragem e de controle. Em [4] foi introduzido o conceito de alcançabilidade fraca ou W-alcançabilidade para SLSM com ruído aleatório, e em [6] a prova do teste foi elaborada usando relações de dualidade com a W-observabilidade e as propriedades de invariância de espaço nulo nas matrizes que compoem a matriz de controlabilidade. Neste artigo, apresentamos uma prova mais simples e direta para este teste, usando somente resultados de algebra linear. Mostramos alguns exemplos numéricos que ilustram este procedimento.

Além disso, formalizamos dois problemas de controle do tipo alcançabilidade (respectivamente controlabilidade), que buscam uma estratégia de controle $u(k)=f(x(k), \theta(0), \ldots, \theta(k))$ que nos permita atingir um alvo $x(\ell)=x_{\ell}$ (respectivamente a origem) num tempo finito $\ell$. Tendo em vista que não há uma metodologia disponível para síntese da função $f$, levantamos algumas possíveis maneiras de avançar na elaboração de um método, apresentando algumas questões em aberto relacionadas com a síntese de controle e W-alcançabilidade. Estas quetões abordam condições testáveis para existência de solução, aproveitando o teste de W-alcançabilidade, e também o problema de obter uma sequência de estados de Markov que, sendo realizada pela cadeia, leve $x$ ao estado desejado.

Organização do artigo: na seção 2 introduzimos a notação utilizada e mostramos alguns resultados prévios como uma extensão do teorema de Cayley-Hamilton. Na seção 3 introduzimos o conceito de $\mathrm{W}$-alcançabilidade, construimos as matrizes de alcançabilidade e mostramos o resultado que verifica a validez do teste. Na seção 4 apresentamos os problemas de controle e questões em aberto relacionadas. Finalmente, na seção 5 apresentamos um exemplo que satisfaz o teste proposto e sua respectiva estratégia de controle.

\section{Preliminares}

Denotemos por $\mathcal{R}^{n}$ o espaço vetorial real de dimensão $n, \mathcal{M}^{n, m}$ o espaço vetorial das matrizes reais de dimensão $n \times m, \mathcal{M}^{n}=\mathcal{M}^{n, n}, \mathcal{M}^{n 0}\left(\mathcal{M}^{n+}\right)$ o cone convexo fechado das matrizes simétricas positiva semidefinidas (o cone aberto das matrizes simétricas positivas definidas). Escrevemos por $U \geq 0(U>0)$ quando $U \in \mathcal{M}^{n 0}\left(U \in \mathcal{M}^{n+}\right)$, por $U^{\prime}$ a matriz transposta de $U$. Representamos por $I_{n}$ a matriz identidade de dimensão $n, 0_{n}$ a matriz nula de dimensão $n$ e $1_{\{\cdot\}}$ o operador indicador.

Denotamos por $\mathcal{C}^{n, m}$ o espaço vetorial formado pela coleção de matrizes $\mathbb{U}=\left\{U_{i}\right\}_{i \in T}$ e para simplificar a notação fazemos $\mathcal{C}^{n}=\mathcal{C}^{n, n} ; \mathcal{C}^{n 0}$ (respectivamente $\mathcal{C}^{n+}$ ) é o subespaço das coleções de matrizes $\mathbb{U}$, com $U_{i} \in \mathcal{M}^{n 0}$ (respectivamente $U_{i} \in \mathcal{M}^{n+}$ ). Consideramos $\theta(k)$ uma cadeia de Markov que assume em cada instante de tempo $k$ um único valor em $T$, com matriz de probabilidades de transição $P=\left[p_{i, j}\right] \in \mathcal{M}^{N}$ e distribuição $\pi_{i}(k)=\operatorname{Prob}(\theta(k)=i)$. Assumindo que a cadeia é ergódica, veja [5], temos que $\pi_{i}(k)>0$, para cada $k \geq 0$ e $i \in T$.

Para $\mathbb{A} \in \mathcal{C}^{n}$ e $\mathbb{B} \in \mathcal{C}^{m, n}$, definimos um sistema linear com saltos markovianos (SLSM's) a tempo discreto da seguinte forma:

$$
\left\{\begin{array}{cl}
x(k+1) & =A_{\theta(k)} x(k)+B_{\theta(k)} u(k), k \geq 0, \\
x(0) & =x_{0} .
\end{array}\right.
$$

Introduzindo as matrizes $\phi(r, s)=A_{\theta(r-1)} A_{\theta(r-2)} \cdots A_{\theta(s)}, r>s$ e $\phi(r, r)=I_{n}$, podemos escrever a solução de (2) como :

$$
x(k)=\phi(k, 0) x_{0}+\sum_{t=0}^{k-1} \phi(k, t+1) B_{\theta(t)} u(t) .
$$

$\mathrm{Na}$ continuação, apresentamos alguns resultados preliminares e definições. Iniciamos definindo as matrizes $\Gamma(r, s)=\sum_{t=s}^{r-1} \phi(r, t+1) B_{\theta(t)} B_{\theta(t)}^{\prime} \phi(r, t+1)^{\prime}$, para $r>s$ e $\Gamma(r, r)=0_{n}$. Isto verifica

$$
\Gamma(r+1, s)=A_{\theta(r)} \Gamma(r, s) A_{\theta(r)}^{\prime}+B_{\theta(r)} B_{\theta(r)}^{\prime} .
$$


Denotando $\Gamma\left(r, s, i_{s}, \ldots, i_{r-1}\right)=\Gamma(r, s) \cdot 1_{\left\{\theta(r)=i_{r}\right\}} \cdot 1_{\left\{\theta(r-1)=i_{r-1}\right\}} \cdots 1_{\left\{\theta(s-1)=i_{s-1}\right\}}$, construímos as matrizes $\mathbb{X}(k)=\left\{X_{i}(k)\right\}_{i \in T}$, onde

$$
X_{i}(k)=E\left\{\Gamma(k, 0) \cdot 1_{\{\theta(k)=i\}}\right\}, k \geq 1 \quad \text { e } \quad X_{i}(0)=0_{n} .
$$

Seguindo [1], definimos para cada $\mathbb{V} \in \mathcal{C}^{n 0}$, o operador $\mathcal{T}_{\mathbb{V}}$, dado por $\mathcal{T}_{\mathbb{V}, i}(\mathbb{U})=\sum_{j=1}^{N} p_{j i} V_{j} U_{j} V_{j}^{\prime}$. Denotemos por $\mathcal{T}_{\mathbb{V}}^{0}(\mathbb{U})=\mathbb{U}$ e $\mathcal{T}_{\mathbb{V}}^{k}(\mathbb{U})=\mathcal{T}_{\mathbb{V}}\left(\mathcal{T}_{\mathbb{V}}^{k-1}(\mathbb{U})\right), k \geq 1$

Lema 2.1. Seja $\mathbb{Q}(k) \in \mathcal{C}^{n 0}$, onde $Q_{i}(k)=\pi_{i}(k) I_{n}$. Então, vale a afirmação:

$$
X_{i}(k+1)=\mathcal{T}_{\mathbb{A}, i}(\mathbb{X}(k))+\mathcal{T}_{\mathbb{B}, i}(\mathbb{Q}(k)), \quad k \geq 0 .
$$

Definindo $\tilde{\mathbb{Q}}(k)=\mathcal{T}_{\mathbb{B}}(\mathbb{Q}(k))$, temos $\mathbb{X}(k)=\sum_{t=0}^{k-1} \mathcal{T}_{\mathbb{A}}^{t}(\tilde{\mathbb{Q}}(k-t-1))$.

Demonstração. Como $\sum_{j=1}^{N} 1_{\{\theta(k)=j\}}=1, k \geq 0$, aplicando (3) e (4) em $X_{i}(k+1)$, obtemos

$$
\begin{aligned}
X_{i}(k+1)= & \sum_{j=1}^{N}\left(A_{j} E\left\{\Gamma(k, 0) \cdot 1_{\{\theta(k+1)=i\}} \cdot 1_{\{\theta(k)=j\}}\right\} A_{j}^{\prime}+B_{j} E\left\{1_{\{\theta(k+1)=i\}} \cdot 1_{\{\theta(k)=j\}}\right\} B_{j}^{\prime}\right), \\
= & \sum_{j=1}^{N} p_{j i} A_{j} E\left\{\Gamma(k, 0) \cdot 1_{\{\theta(k)=j\}}\right\} A_{j}^{\prime}+\sum_{j=1}^{N} p_{j i} \pi_{j}(k) I_{n} B_{j} B_{j}^{\prime}, \\
& \sum_{j=1}^{N} p_{j i} A_{j} X_{j}(k) A_{j}^{\prime}+\sum_{j=1}^{N} p_{j i} B_{j} Q_{j}(k) B_{j}^{\prime}=\mathcal{T}_{\mathbb{A}, i}(\mathbb{X}(k))+\mathcal{T}_{\mathbb{B}, i}(\mathbb{Q}(k)) .
\end{aligned}
$$

A outra relação é consequência imediata desta relação. Isto completa a prova do lema.

Se escrevemos $V=\left[v_{1} \vdots v_{2} \vdots \ldots \vdots v_{n}\right] \in \mathcal{M}^{n}$ e $\mathbb{U} \in \mathcal{C}^{n}$, de [1], introduzimos os operadores lineares e invertíveis, $\varphi(V)=\left[v_{1}, \ldots, v_{n}\right]^{\prime}$ e $\hat{\varphi}(\mathbb{U})=\left[\varphi\left(U_{1}\right)^{\prime}, \ldots, \varphi\left(U_{N}\right)^{\prime}\right]^{\prime}$. Seguindo a proposição $4\left(\right.$ a) de $[1]$, temos que $\hat{\varphi}\left(\mathcal{T}_{\mathbb{A}}(\mathbb{U})\right)=\mathcal{A} \hat{\varphi}(\mathbb{U})$, onde $\mathcal{A}$ é

$$
\mathcal{A}=\left[\begin{array}{cccc}
p_{11} A_{1} \otimes A_{1} & p_{21} A_{2} \otimes A_{2} & \cdots & p_{N 1} A_{N} \otimes A_{N} \\
p_{12} A_{1} \otimes A_{1} & p_{22} A_{2} \otimes A_{2} & \cdots & p_{N 2} A_{N} \otimes A_{N} \\
\vdots & \vdots & \ddots & \vdots \\
p_{1 N} A_{1} \otimes A_{1} & p_{2 N} A_{2} \otimes A_{2} & \cdots & p_{N N} A_{N} \otimes A_{N}
\end{array}\right] \in \mathcal{M}^{n^{2} N},
$$

Usando esta matriz, apresentamos uma extensão ao Teorema de Cayley-Hamilton, vide [2].

Lema 2.2. Sejam $\mathbb{A}, \mathbb{U} \in \mathcal{M}^{n}$ e $k \geq n^{2} N$. Então existem $\beta_{1}, \ldots, \beta_{n} \in \mathcal{R}$, que verifiquem

$$
\mathcal{T}_{\mathbb{A}}^{k}(\mathbb{U})=\beta_{1} \mathcal{T}_{\mathbb{A}}^{n^{2} N-1}(\mathbb{U})+\beta_{2} \mathcal{T}_{\mathbb{A}}^{n^{2} N-1}(\mathbb{U})+\cdots+\beta_{n^{2} N} \mathcal{T}_{\mathbb{A}}^{0}(\mathbb{U}) .
$$

Demonstração. Como $\mathcal{A} \in \mathcal{M}^{n^{2} N}$, pelo Teorema de Cayley-Hamilton existem $\beta_{1}, \beta_{2}, \cdots, \beta_{n^{2} N}$ tais que $\mathcal{A}^{k}=\beta_{1} \mathcal{A}^{n^{2} N-1}+\beta_{2} \mathcal{A}^{n^{2} N-2}+\cdots+\beta_{n^{2} N} I_{n^{2} N}$. Multiplicando por $\hat{\varphi}(\mathbb{U})$, obtemos $\mathcal{A}^{k} \hat{\varphi}(\mathbb{U})=\beta_{1} \mathcal{A}^{n^{2} N-1} \hat{\varphi}(\mathbb{U})+\beta_{2} \mathcal{A}^{n^{2} N-2} \hat{\varphi}(\mathbb{U})+\cdots+\beta_{n^{2} N} I_{n^{2} N} \hat{\varphi}(\mathbb{U})$. Como $\mathcal{A}^{j} \hat{\varphi}(\mathbb{U})=\hat{\varphi}\left(\mathcal{T}_{\mathbb{A}}^{j}(\mathbb{U})\right)$, temos $\hat{\varphi}\left(\mathcal{T}_{\mathbb{A}}^{k}(\mathbb{U})\right)=\beta_{1} \hat{\varphi}\left(\mathcal{T}_{\mathbb{A}}^{n^{2} N-1}(\mathbb{U})\right)+\beta_{2} \hat{\varphi}\left(\mathcal{T}_{\mathbb{A}}^{n^{2} N-2}(\mathbb{U})\right)+\cdots+\beta_{n^{2} N} \hat{\varphi}\left(\mathcal{T}_{\mathbb{A}}^{0}(\mathbb{U})\right)$. Finalmente da linearidade e invertivilidade do operador $\hat{\varphi}$, verificamos (5).

\section{W-alcançabilidade}

Nesta seção, apresentamos o conceito de W-alcançabilidade e um teste computacional, e derivamos uma demonstração direta de que este teste é uma condição necessária e suficiente para que o sistema seja W-alcançável. Para construir este teste, definamos $\mathbb{W}(k) \in \mathcal{C}^{n, n k}$, onde $W_{i}(k)$ é

$$
W_{i}(k)=\left[\begin{array}{lllllll}
X_{i}(1) & \vdots & X_{i}(2) & \vdots & \cdots & \vdots & X_{i}(k)
\end{array}\right], \quad i \in T .
$$


Observação 3.1. Consequência de (6), vale a afirmação: $\operatorname{Se} \operatorname{Ker}\left(W_{i}(k) W_{i}(k)^{\prime}\right) \neq\{0\}$, então $\operatorname{Ker}\left(X_{i}(t)\right) \neq\{0\}$, para cada $t=1,2, \ldots, k$.

Um resultado que possibilita tornar finito o número de iterações do teste de $\mathrm{W}$-alcançabilidade está relacionado com o seguinte lema.

Lema 3.1. Seja $i \in T$ e $k>n^{2} N$, vale a afirmação: $S e \operatorname{ker}\left(W_{i}\left(n^{2} N\right) W_{i}\left(n^{2} N\right)^{\prime}\right) \neq\{0\}$, então $\operatorname{ker}\left(W_{i}(k) W_{i}(k)^{\prime}\right) \neq\{0\}$. Equivalentemente, se posto $\left(W_{i}\left(n^{2} N\right)^{\prime}\right)<n$, então posto $\left(W_{i}(k)^{\prime}\right)<n$.

Demonstração. Lembremos do lema 2.1 que $X_{i}\left(n^{2} N\right)=\mathcal{T}_{\mathbb{A}, i}^{n^{2} N-1}(\tilde{\mathbb{Q}}(0))+\cdots+\mathcal{T}_{\mathbb{A}, i}^{0}\left(\tilde{\mathbb{Q}}\left(n^{2} N-1\right)\right)$, pela observação 3.1 existe $v \in \mathcal{R}^{n}$, com $v \neq 0$ que verifica $X_{i}\left(n^{2} N\right) v=0$. Portanto

$$
\mathcal{T}_{\mathbb{A}, i}^{k}(\tilde{\mathbb{Q}}(t)) v=0, \quad k=0,1, \ldots, n^{2} N-1 \text { e } t=0,1, \ldots, n^{2} N-1 .
$$

Assumindo $k=n^{2} N+1, \ldots, 2 n^{2} N$, do lema 2.1 temos $X_{i}(k)=\mathcal{T}_{\mathbb{A}, i}^{k-1}(\tilde{\mathbb{Q}}(0))+\cdots+\mathcal{T}_{\mathbb{A}, i}^{0}(\tilde{\mathbb{Q}}(k-1))$ e aplicando o lema 2.2 , escrevemos $\mathcal{T}_{\mathbb{A}, i}^{t}(\tilde{\mathbb{Q}}(k-t-1))$, para $t=n^{2} N, \ldots, k-1$, como:

$$
\mathcal{T}_{\mathbb{A}, i}^{t}(\tilde{\mathbb{Q}}(k-t-1))=\beta_{1}^{t} \mathcal{T}_{\mathbb{A}, i}^{n^{2} N-1}(\tilde{\mathbb{Q}}(k-t-1))+\cdots+\beta_{n^{2} N}^{t} \mathcal{T}_{\mathbb{A}, i}^{0}(\tilde{\mathbb{Q}}(k-t-1)) .
$$

Desta relação e as equações (5) e (7), obtemos

$$
\begin{aligned}
X_{i}(k) v & =\left(\mathcal{T}_{\mathbb{A}}^{0}(\tilde{\mathbb{Q}}(k-1))+\mathcal{T}_{\mathbb{A}}^{1}(\tilde{\mathbb{Q}}(k-2))+\cdots+\mathcal{T}_{\mathbb{A}}^{n^{2} N-1}\left(\tilde{\mathbb{Q}}\left(k-n^{2} N\right)\right)\right) v \\
& +\left(\beta_{1}^{n^{2} N} \mathcal{T}_{\mathbb{A}}^{n^{2} N-1}\left(\tilde{\mathbb{Q}}\left(k-n^{2} N-1\right)\right)+\cdots+\beta_{n^{2} N}^{n^{2} N} \mathcal{T}_{\mathbb{A}}^{0}\left(\tilde{\mathbb{Q}}\left(k-n^{2} N-1\right)\right)\right) v \\
& +\cdots \\
& +\left(\beta_{1}^{k-1} \mathcal{T}_{\mathbb{A}}^{n^{2} N-1}(\tilde{\mathbb{Q}}(0))+\cdots+\beta_{n^{2} N}^{k-1} \mathcal{T}_{\mathbb{A}}^{0}(\tilde{\mathbb{Q}}(0))\right) v=0,
\end{aligned}
$$

isto mostra que $X_{i}(k) v=0$. Portanto $\operatorname{ker}\left(W_{i}(k) W_{i}(k)^{\prime}\right) \neq\{0\}, k=n^{2} N+1, \ldots, 2 n^{2} N$. Em particular $\operatorname{ker}\left(W_{i}\left(2 n^{2} N\right) W_{i}\left(2 n^{2} N\right)^{\prime}\right) \neq\{0\}$, pelo mesmo procedimento $\operatorname{ker}\left(W_{i}(k) W_{i}(k)^{\prime}\right) \neq\{0\}$, $k=2 n^{2} N+1, \ldots, 4 n^{2} N$. Podemos mostrar por indução que $\operatorname{ker}\left(W_{i}(k) W_{i}(k)^{\prime}\right) \neq\{0\}$ para qualquer $k>n^{2} N$. Lembremos que $\operatorname{ker}\left(W W^{\prime}\right)=\operatorname{ker}\left(W^{\prime}\right)$, para qualquer $W \in \mathcal{M}^{n, p}$, então vale a afirmação: Se $\operatorname{ker}\left(W_{i}\left(n^{2} N\right)^{\prime}\right) \neq\{0\}$ então $\operatorname{ker}\left(W_{i}(k)^{\prime}\right) \neq\{0\}$. Logo, pelo teorema fundamental da algebra linear, veja [2], vale a afirmação: $\operatorname{Se~} \operatorname{posto}\left(W_{i}\left(n^{2} N\right)\right)<n$ então $\operatorname{posto}\left(W_{i}(k)\right)<n$.

Definição 3.1. O sistema (2) é $W$-alcançável se e somente se existe $\ell \geq 1$ que verifique

$$
E\{\Gamma(\ell, 0) \mid \theta(0)=i\}>0, \text { para cada } i \in \mathbb{S} .
$$

$A$ coleção de matrizes de $W$-alcançabilidade é $\mathbb{W}\left(n^{2} N\right)=\left\{W_{1}\left(n^{2} N\right), \ldots, W_{N}\left(n^{2} N\right)\right\}$.

Observação 3.2. Se o sistema é $W$-alcançável então $X_{i}(k)>0$, para $k \geq \ell$. Para mostrar isso basta considerar $\Gamma(k, 0)=\Gamma(k, \ell)+\Gamma(\ell, 0)$ e usar as definições as propriedades de esperança condicionada, vide [5] e as definições dadas em (8) e (4).

A seguir apresentamos o teste de W-alcançabilidade encontrado em [6].

Teorema 3.1. [Teste de W-alcançabilidade] O sistema (2) é $W$-alcançável se e somente se todas as matrizes de $W$-alcançabilidade tem posto completo.

Uma prova para o Teorema 3.1 é encontrada em [6], sendo bastante indireta. Mais precisamente, constroi-se um sistema auxiliar (que por sua vez envolve uma cadeia de Markov com taxas "reversas") juntamente uma função de custo auxiliar; em seguida, identifica-se a observabilidade fraca do sistema auxiliar com a alcançabilidade fraca, o que permite a adaptação de um teste anteriormente apresentado para W-observabilidade. Vide [6] [Teorema 2].

Neste trabalho desenvolvemos uma demonstração direta, que se utiliza apenas de ferramentas de álgebra linear e dos resultados que apresentamos anteriormente nesta seção. 
Prova do Teorema $3.1(\Rightarrow)$ Dado $i \in T$, considerando $\ell \geq 1$ que satisfaz (8), pela observação 3.2 temos $X_{i}(k)>0$. Se $k=n^{2} N$, então $X_{i}\left(n^{2} N\right)>0$ e

$$
v^{\prime} W_{i}\left(n^{2} N\right) W_{i}\left(n^{2} N\right)^{\prime} v=v^{\prime} X_{i}(1) X_{i}(1)^{\prime} v+\cdots+v^{\prime} X_{i}\left(n^{2} N\right) X_{i}\left(n^{2} N\right)^{\prime} v>0 .
$$

Para qualquer $W \in \mathcal{M}^{n, p}$ vale que $W>0$ implica em $\operatorname{ker}(W)=\{0\}$ ou, equivalentemente, $\operatorname{posto}\left(W^{\prime}\right)=n$, de maneira que de (9) vem

$$
\operatorname{ker}\left(W_{i}\left(n^{2} N\right) W_{i}\left(n^{2} N\right)^{\prime}\right)=\operatorname{ker}\left(W_{i}\left(n^{2} N\right)^{\prime}\right)=\{0\} \Leftrightarrow \operatorname{posto}\left(W_{i}\left(n^{2} N\right)\right)=n .
$$

Se $k<n^{2} N$, então $X_{i}(k)>0$ e $W_{i}(k) W_{i}(k)^{\prime}>0$. Como $W_{i}\left(n^{2} N\right) W_{j}\left(n^{2} N\right)^{\prime}=W_{i}(k) W_{i}(k)^{\prime}+$ $\cdots+X_{i}\left(n^{2} N\right) X_{i}\left(n^{2} N\right)^{\prime}$, então $\operatorname{ker}\left(W_{i}\left(n^{2} N\right) W_{i}\left(n^{2} N\right)^{\prime}\right)>0$ e de $(10)$ temos que $W_{i}\left(n^{2} N\right)$ tem posto completo. Similarmente, se $k>n^{2} N$ então $\operatorname{ker}\left(W_{i}(k) W_{i}(k)^{\prime}\right)=\operatorname{ker}\left(W_{i}(k)^{\prime}\right)=\{0\}$, então pela recíproca do Lema 3.1 temos $\operatorname{ker}\left(W_{i}\left(n^{2} N\right)^{\prime}\right)=\{0\}$, e de $(10)$ verificamos que $W_{i}\left(n^{2} N\right)$ tem posto completo.

$(\Leftarrow)$ Seja $i \in T$ tal que posto $\left(W_{i}\left(n^{2} N\right)\right)=n$, isto implica que $\operatorname{Ker}\left(W_{i}\left(n^{2} N\right) W_{i}\left(n^{2} N\right)^{\prime}\right)=\{0\}$ e portanto $W_{i}\left(n^{2} N\right) W_{i}\left(n^{2} N\right)^{\prime}>0$. Como $i$ foi escolhido arbitrariamente, existe $t_{i} \leq n^{2} N$ tal que $X_{i}\left(t_{i}\right)>0$. Em geral verifica-se $X_{i}(t)>0$ para cada $t \geq t_{i}$. Se escolhemos $p=\max \left\{t_{i}, i \in T\right\}$ então $X_{i}(p)>0$ e $E\left\{\Gamma(p+\ell, p) \cdot 1_{\{\theta(p)=i\}}\right\}>0, \quad \ell \geq 0$ e $i \in T$. Como o SLSM em questão (2) é homogêneo no tempo, temos $E\left\{\Gamma(\ell, 0) \cdot 1_{\{\theta(0)=i\}}\right\}=E\left\{\Gamma(p+\ell, p) \cdot 1_{\{\theta(p)=i\}}\right\}$, portanto a equação acima leva a $E\left\{\Gamma(\ell, 0) \cdot 1_{\{\theta(0)=i\}}\right\}>0$. Relembrando que assumimos $\pi_{i}(0)>0$, completa-se a prova notando que $E\left\{\Gamma(\ell, 0) \cdot 1_{\{\theta(0)=i\}}\right\}=E\{\Gamma(\ell, 0) \mid \theta(0)=i\} \cdot \pi_{i}(0)$.

\section{Problemas de controle e questões em aberto}

Similarmente ao caso de sistemas lineares determinísticos, para SLSM podemos definir problemas de controle associados com a estrutura do sistema, como por exemplo o problema de alcançabilidade que, resumidamente, visa que $x$ atinja um "alvo". Claro, é possível definir diversos problemas dependendo do critério adotado para considerar que o alvo foi atingido (em média, com alguma probabilidade, visita a uma vizinhança, em tempo finito ou infinito, etc.); algumas variações foram consideradas na literatura, contudo aquelas definidas abaixo foram muito pouco exploradas no contexto de SLSM, apesar de terem um considerável apelo para aplicações.

Problema 1 (Problema de Controlabilidade). Encontrar uma estratégia de controle da forma $u(k)=f(x(k), \theta(k)), k=\{0,1, \ldots, \ell-1\}$ tal que, assumindo $x_{0} \neq 0$, leve a $\operatorname{Prob}(x(\ell)=0)>0$, sendo $\ell$ um horizonte de tempo pré-especificado.

Problema 2 (Problema de Alcançabilidade). Encontrar uma estratégia de controle da forma $u(k)=f(x(k), \theta(k)), k=\{0,1, \ldots, \ell-1\}$ tal que, assumindo $x_{0}=0$, leve a $\operatorname{Prob}\left(x(\ell)=x_{1}\right)>0$, sendo $\ell$ um horizonte de tempo pré-especificado.

Não existe uma metodologia que permita encontrar a solução dos problemas acima para SLSM, aproveitando a estrutura linear e as propriedades da cadeia de Markov (existem propostas para sistemas com chaveamento geral e para sistemas variantes no tempo, mas são de difícil implementação devido a "alta dimensionalidade" / elevado esforço computacional). Associado a síntese de controle, temos algumas questões importantes que, na extensão do nosso conhecimento, estão em aberto para SLSM.

- Demonstração de que W-alcançabilidade é uma condição necessária para existência de solução para os problemas de alcançabilidade e controlabilidade. Temos bons indícios de que esta conjectura é verdadeira, através de estudo de casos numéricos.

- Verificar se W-alcançabilidade é uma condição suficiente para existência de solução para os problemas de alcançabilidade e controlabilidade. 
- Verificar o papel de W-alcançabilidade (e do teste do Teorema 3.1) no levantamento de uma sequência de estados de Markov $i_{1}, \ldots, i_{\ell}$ que, sendo realizada pela cadeia, permite a síntese de uma ação de controle via o seguinte sistema:

$$
x_{1}-\phi(\ell, 0)=M_{\ell} U_{\ell}
$$

sendo $M_{\ell}=\left[B_{\theta(\ell-1)} \vdots \phi(\ell, \ell-1) \vdots \ldots, \vdots, \phi(\ell, 1) B_{\theta(0)}\right]$ e $U_{\ell}=\left[u(\ell-1)^{\prime}, \ldots, u(0)^{\prime}\right]^{\prime}$. É interessante comentar que é possível procurar sequências da forma acima de maneira exaustiva, contudo isso tende a ser extremamente pesado computacionalmente uma vez que o número de (diferentes) realizações cresce de acordo com $N^{\ell}$ sendo $\ell$ o horizonte de tempo, e para cada sequência é preciso verificar a existência de solução para (11)

- Escrever o problema de encontrar a sequência $i_{0}, i_{1}, \ldots, i_{\ell}-1$ de maneira separada em estágios de tempo, buscando possibilitar o uso de técnicas de programação dinâmica. Consideramos pouco provável que se possa definir um problema com uma função valor associada, da forma tradicional, contudo pode haver formalizações mais gerais (por exemplo multiobjetivo) que permita enquadrar o problema na forma de programação dinâmica.

- Estudar as questões acima considerando variações da noção de W-alcançabilidade, por exemplo inserindo restrições nos estados da cadeia.

- Extender os resultados para SLSM a tempo contínuo e relacionar os resultados com um problema de alcançabilidade estocástica para sistemas hibrido, trabalhados em [7].

\section{Exemplos numérico}

Nesta seção usaremos o teste do Teorema 3.1 para verificar se um sistema tipo (2) é W-alcançável. Também apresentamos soluções para o problema de controlabilidade da Seção 4.

Exemplo: Consideremos um SLSM $\operatorname{com} T=\{1,2\}$,

$$
A_{1}=\left[\begin{array}{ll}
1 & 0 \\
0 & 1
\end{array}\right], A_{2}=\left[\begin{array}{rr}
\cos \alpha & \sin \alpha \\
-\sin \alpha & \cos \alpha
\end{array}\right], B_{1}=\left[\begin{array}{l}
1 \\
0
\end{array}\right], B_{2}=\left[\begin{array}{l}
0 \\
0
\end{array}\right], P=\left[\begin{array}{ll}
0.5 & 0.5 \\
0.5 & 0.5
\end{array}\right]
$$

e a distribuição inicial $\pi=[0.5,0.5]$. Para $\alpha=20^{\circ}$, calculamos as matrizes de W-alcançabilidade e verificamos que estas matrizes tem posto completo, portanto o sistema é W-alcançável. Neste

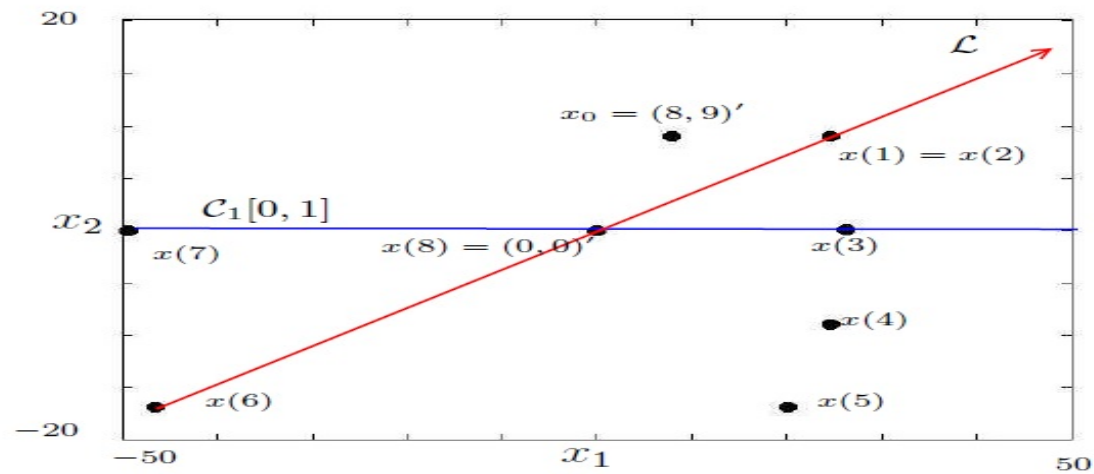

Figura 1: Diagrama de fase com $x_{0}=(8,9)$ para realização da cadeia $\{1,1,2,2,2,1,2,1\}$.

exemplo, a estratégia de controle foi concebida para levar o estado $x(k+1)$ ao subespaço "intermediário" $\mathcal{L}=\left\{(x, y) \in \mathbb{R}^{2}:(x, y)=(t \cos \alpha, t \operatorname{sen} \alpha), t \in \mathbb{R}\right\}$ tal que $\mathcal{C}_{1}=A_{2} \mathcal{L}$, o que é possível sempre que $\theta(k)=1$; em seguida simplesmente se faz $u(k+1)=0$ e $u(k+2)=-x_{1}(k)$, o 
que leva o estado até a origem se $\theta(k+1)=2$ e $\theta(k+2)=1$. Veja na Figura 1 a trajetória de $x(k)$ associada a realização $\{1,1,2,2,2,1,2,1\}$ da cadeia, garantimos que o estado é levado a origem (resolvendo o problema de controlabilidade) verificando (11), após a sequência 1,2,1. Neste exemplo, garantimos $\operatorname{Prob}(x(\ell)=0) \geq \operatorname{Prob}(\theta(0)=1, \theta(1)=2, \theta(2)=1)>0$, o que está de acordo com as conjecturas da Seção 4 (W-alcançabilidade condição para existência de solução).

\section{Conclusões}

Neste artigo, apresentamos uma demonstração direta para o teste de W-alcançabilidade, como enunciado no Teorema 3.1. Também apresentamos problemas de controlabilidade e alcançabilidade e levantamos questões em aberto relacionando estes problemas com a noção de $\mathrm{W}$-alcançabilidade. Finalizamos com um exemplo numérico ilustrativo.

\section{Referências}

[1] O. L. V. Costa, M. D. Fragoso and R. P. Marques. Discrete-time markovian jump linear systems (Springer-Verlag, eds.) pp.1-27, New York, 2005.

[2] J. P. Hspanha. Linear systems theory (Princeton University Press, eds.) pp.95-109, New Jersey, 2009.

[3] P. J. Antsaklis and A. N. Michel. Linear systems (Birkhauser, eds.) pp.214-246, Boston, 2006.

[4] A. L. P. Manfrim, "O conceito de estabilizabilidade fraca para sistemas lineares com saltos markovianos", Dissertação de Mestrado, ICMC-USP, 2006.

[5] E. Cinlar, Introduction to stochastic processes (Prentice-Hall, eds.) pp. 106-138, New Jersey, 1975.

[6] A. R. R. Narváez and E. F. Costa. On the controllability of continuos-time markov jump linear system, IFAC 18th World Congress of the International Federation of Automatic Control, Milano, 2011.

[7] L. M. Bujorianu. Stochastic reachability analysis of hibrid systems (Springer, eds.) pp.87134, London, 2012. 